\title{
Baixa Estatura na Doença Renal Crônica: Fisiopatologia e Tratamento com Hormônio de Crescimento
}

\begin{abstract}
RESUMO
O atraso no crescimento é freqüente e grave em crianças com doença renal crônica (DRC). Vários fatores contribuem para o comprometimento do crescimento nestas crianças, incluindo as alterações no eixo hormônio de crescimento $(\mathrm{GH})$ - insulin-like growth factor 1 (IGF-1), desnutrição, acidose, doença renal óssea e uso de corticóides. Em crianças com DRC, o tratamento do atraso no crescimento é difícil em virtude da presença de doenças associadas que necessitem de adequado tratamento médico. Apesar de as evidências a respeito da segurança e de a eficácia do $\mathrm{GH}$ nesta população, este tratamento ainda é pouco utilizado. Esta revisão mostra o impacto, as causas e o tratamento do atraso no crescimento em crianças com DRC. (Arq Bras Endocrinol Metab 2008; 52/5:783-791)
\end{abstract}

Descritores: Falência renal crônica; Crescimento; Hormônio de crescimento; Corticóides; Diálise renal; Transplante renal

\section{ABSTRACT}

Short Stature in Chronic Kidney Disease: Physiopathology and Treatment with Growth Hormone.

Growth failure is frequent and a clinically important issue in children with chronic kidney disease (CKD). Many factors contribute to impaired growth in these children, including abnormalities in the growth hormone $(\mathrm{GH})$ - insulinlike growth factor 1 (IGF-1) axis, malnutrition, acidosis, renal bone disease and glucocorticoid associated treatment. The management of growth failure in children with CKD is complicated by the presence of other-disease related complications requiring medical intervention. Despite evidence of $\mathrm{GH}$ efficacy and safety in this population, this therapy is still underutilized. This review shows the impact, the causes and the treatment of growth failure in children with CKD. (Arq Bras Endocrinol Metab 2008; 52/5:783-791)

Keywords: Chronic kidney failure; Growth; Growth hormone; Corticoids; Kidney dialysis; Kidney transplantation

\section{DEFININDO INSUFICIÊNCIA RENAL CRÔNICA E DOENÇA RENAL CRÔNICA}

$\mathrm{H}$ ISTORICAMENTE, O TERMO INSUFICIÊNCIA renal crônica (IRC) tem sido usado para descrever pacientes com ritmo de filtração glomerular (RFG) menor que $75 \mathrm{~mL} / \mathrm{min} / 1,73 \mathrm{~m}^{2}$ de área corporal (1). Mais recentemente, a National Kidney Foundation (NKF) introduziu o termo doença renal crônica (DRC), bem como a classificação do quadro, a fim de propiciar a detecção precoce, retardar a progressão e prevenir complicações relacionadas. Essa classificação descreve cinco estágios da DRC considerando a presença de lesão revisão

Josenilson Campos de Oliveira

ADRIANA A. SIVIERO-MIACHON

Angela Maria Sinola-Castro

Vera Maria Santoro Belangero

GIL GuerRa-Junior

Clínica Médica da Faculdade de Ciências Médicas (FCM) da Universidade Estadual de Campinas (Unicamp), Campinas, SP, Brasil (JCO); Programa de Pós-graduação do Curso de Pediatria da Escola Paulista de Medicina (EPM) da Universidade Federal de São Paulo (Unifesp), São Paulo, SP, Brasil (AASM); Unidade de Endocrinologia Pediátrica, Departamento de Pediatria da EPM-Unifesp, São Paulo, SP, Brasil (AMSC); Unidade de Nefrologia Pediátrica, Departamento de Pediatria da FCM-Unicamp, Campinas, SP, Brasil (VMSB); Unidade de Endocrinologia Pediátrica, Departamento de Pediatria da FCM-Unicamp, Campinas, SP, Brasil (GGJ).

Recebido em 21/4/2008

Aceito em 24/4/2008 
renal e o grau de comprometimento funcional, independente do diagnóstico da doença de base (Tabela 1 ). Define-se a lesão renal pela presença de qualquer marcador de doença renal, considerando dados clínicos, como antecedente de agenesia renal unilateral, e/ou alterações laboratoriais, como hematúria e microalbuminúria. O comprometimento funcional é determinado pelo RFG, calculado por fórmulas, a partir do valor da creatinina sérica ou do valor estimado pela coleta do clearance de creatinina. Importante ressaltar que para se incluir dentro do diagnóstico de DRC, as alterações devem ter duração maior que três meses. Os valores do RFG apresentados na Tabela 1 são aplicáveis às crianças maiores de 2 anos, e para as menores de 2 anos pode-se utilizar a perda de $50 \%$ do RFG definido como normal, como a fase inicial da DRC (1).

Tabela 1. Classificação dos estágios da doença renal crônica, de acordo com a National Kidney Foundation (1).

\begin{tabular}{|c|c|c|}
\hline Estágio & $\begin{array}{c}\text { RFG (mL/ } \\
\left.\min / 1,73 \mathrm{~m}^{2}\right)\end{array}$ & Descrição \\
\hline 1 & $\geq 90$ & $\begin{array}{c}\text { Lesão renal detectada por } \\
\text { história ou alterações } \\
\text { laboratoriais }\end{array}$ \\
\hline 2 & $60-89$ & $\begin{array}{c}\text { Lesão renal com redução leve } \\
\text { do RFG }\end{array}$ \\
\hline 3 & $30-59$ & Redução moderada do RFG \\
\hline 4 & $15-29$ & Redução grave do RFG \\
\hline 5 & < 15 (ou diálise) & Insuficiência renal \\
\hline
\end{tabular}

RFG = ritmo de filtração glomerular; valores aplicáveis a crianças com idade $\geq$ 2 anos.

Nos Estados Unidos, 300 mil pessoas têm DRC estágio $5 ; 400$ mil estágio $4 ; 8$ milhões estágio $3 ; 5$ milhões estágio 2; e 6 milhões têm DRC com TFG normal ou alta. Este alto número de pessoas com DRC com moderada ou leve alteração na TFG poderão ter IRC nos próximos dez anos, e as crianças com DRC são os potenciais adultos com DRC grave ou IRC. Para cada caso diagnosticado de DRC no estágio 5, deve existir 50 casos de DCR entre os estágios 1 e 4 (2). Deve-se salientar que, embora a incidência da DRC na infância seja 50 vezes menos freqüente que a do adulto, a DRC é devastadora na infância levando a repercussões graves no desenvolvimento físico, neurológico, psíquico e social. De acordo com os dados da North American Pediatric Renal Transplant Cooperative Study (NAPRTCS), as doenças que mais levam à IRC na infância são as doenças associadas a malformações do trato urinário, as doenças glomerulares e as doenças hereditárias (3).

\section{ATRASO NO CRESCIMENTO EM CRIANÇAS E ADOLESCENTES COM DOENÇA RENAL CRÔNICA}

Em crianças portadoras de DRC, o atraso no crescimento é um problema freqüente e importante. Os pacientes em tratamento conservador, dialítico ou mesmo após o transplante apresentam redução na velocidade de crescimento, que tende a ser mais intensa conforme o estágio mais avançado da doença renal (4). O atraso no crescimento nessa população tem etiologia multifatorial, refletindo anormalidades no eixo GH/IGF/ IGFBP (5), bem como distúrbios metabólicos e nutricionais, que exigem manuseio adequado para melhorar o crescimento (6). Atingir a altura adulta normal é um dos aspectos indicativos do sucesso no tratamento (7).

Nas duas últimas décadas, o hormônio de crescimento recombinante humano (hrGH) tem sido usado como estratégia para melhorar a altura final de crianças com IRC (8). No entanto, observa-se que este tratamento está restrito a apenas 6,5\% dos pacientes transplantados e 15,5\% daqueles em diálise, o que claramente demonstra a sua subutilização nos pacientes com DRC (9-11).

Em 2007, no Reino Unido, Lewis e cols. (11) mostraram que $41 \%$ dos pacientes em diálise e $29 \%$ dos pacientes transplantados apresentavam altura abaixo do terceiro percentil. Os fatores, como gênero masculino, menor idade e maior período em diálise, já foram associados a maiores perdas em altura (6). Os dados do NAPRTCS também reforçam que o atraso no crescimento de pacientes com DRC é problema comum e dificilmente revertido, apesar da adequação do tratamento conservador ou da dose de diálise (3).

Aproximadamente um terço das 5.972 crianças com DRC, incluídas no relatório de 2005 do NAPRTCS $(3,4)$, apresentaram atraso no crescimento ( $\mathrm{z}$-escore da altura $\leq-1,88)$ na entrada no estudo. $O$ atraso no crescimento foi mais freqüente quanto menor a idade: $58 \%$ dos pacientes de 0 a 1 ano, $43 \%$ de 2 a 5 anos, $33 \%$ de 6 a 12 anos e $23 \%$ dos maiores de 12 anos. Existiu correlação entre a perda de função renal em qualquer grau e o comprometimento em altura, no entanto, cerca de $20 \%$ daqueles com RFG relativamente adequado (50 a $75 \mathrm{~mL} / 1,73 \mathrm{~m}^{2}$ ) apresentaram perda significativa em altura. 
Ao final do primeiro mês de diálise, entre 3.910 pacientes, o z-escore médio para a altura foi de $-1,66$. Similarmente ao observado no grupo registrado com DRC, os pacientes mais jovens apresentaram perdas mais importantes no crescimento: $\mathrm{z}=-2,54$ de 0 a $\mathrm{l}$ ano, $-1,98$ de 2 a 5 anos, $-1,69$ de 6 a 12 anos e $-1,30$ nos maiores de 12 anos. As análises realizadas seis a doze meses após o início da diálise não mostraram melhora no z-escore para a altura $(3,4)$. Apesar de a literatura apresentar a perda de 0,4 a 0,8 desvio-padrão no z-escore da altura para cada ano em hemodiálise, Tom e cols. (12), intensificando o suporte nutricional de 12 crianças em hemodiálise por cerca de quatro anos, observaram o aumento de 0,3 desvio-padrão no z-escore da altura para cada ano.

Nos dados do NAPRTCS $(3,4)$, o comprometimento da altura também foi evidente no período do transplante renal, porquanto os mais jovens apresentaram os déficits mais importantes. Dos 8.141 pacientes transplantados de 1987 a 2004, o z-escore médio encontrado foi de $-1,98$ para pacientes de 0 a 1 ano, $-2,34$ de 2 a 5 anos, $-2,07$ de 6 a 12 anos e $-1,49$ entre os maiores de 12 anos. Após o transplante, pacientes entre 0 e 5 anos apresentaram melhora no crescimento, especialmente nos dois primeiros anos, enquanto os maiores de 12 anos não obtiveram incremento na altura. Notou-se, também, diferença do z-escore médio da altura no momento do transplante entre os pacientes avaliados em 1987 $(-2,40)$ e em $2004(-1,40)$. Tal melhora já deve ser reflexo do aumento do número de pacientes que usaram o hrGH antes do transplante renal. Outros fatores envolvendo o crescimento após transplante renal foram observados nos dados do NAPPRTCS de $2005(3,4)$ : a) incremento de 0,17 desvio-padrão na altura para cada $1 \mathrm{mg} / \mathrm{dL}$ de redução na concentração da creatinina sérica; b) os caucasianos tiveram melhor recuperação no crescimento quando comparados aos afro-americanos e hispânicos; c) a corticoterapia em dias alternados correlacionou-se a maiores ganhos em altura quando comparada à contínua.

\section{CAUSAS E FISIOPATOLOGIA DO ATRASO NO CRESCIMENTO NA DOENÇA RENAL CRÔNICA}

O atraso no crescimento na DRC tem origem multifatorial. Há vários anos, a desnutrição calórico-protéica tem sido referida como a principal causa de atraso no crescimento de crianças e adolescentes com DRC. A anorexia está presente na maioria destes casos, sendo atribuída aos efeitos da uremia, do paladar alterado, das náuseas e dos vômitos, do estresse psicológico e de fatores endógenos, como a orexina ghrelina e a anorexina leptina (13-18). Vários outros fatores podem contribuir para o atraso no crescimento na DRC, incluindo idade de início da doença renal, etiologia da doença renal, presença de anemia, de acidose metabólica e de osteodistrofia renal, além do próprio potencial genético de crescimento $(2,10)$. No entanto, paulatinamente, as alterações no eixo GH/IGF se tornaram importantes, particularmente em pacientes em corticoterapia $(5,7)$.

Em virtude de cerca da metade da altura final adulta ser adquirida nos dois primeiros anos de vida, o impacto no crescimento é tanto maior quanto mais cedo a doença renal se inicia (2). No entanto, nos dois primeiros anos de vida, desde que se priorize a oferta calórica e protéica nos mesmos moldes que para crianças saudáveis, o crescimento da criança com DRC pode ser normal, tendo-se sempre em mente que nunca se deve restringir a oferta alimentar para adiar a indicação de terapia renal substitutiva. Já durante a puberdade, outra fase de grande crescimento, os pacientes com DRC iniciada na infância tendem a apresentar estirão puberal atrasado e de pequena amplitude. Enquanto as concentrações de gonadotrofinas encontram-se elevadas por causa da redução no cleveance renal, a secreção do hormônio luteinizante (LH) está substancialmente diminuída em pacientes com DRC em comparação aos adolescentes normais. Conseqüentemente, nos meninos, a concentração plasmática de testosterona está diminuída e a testosterona livre encontra-se ainda menor em virtude do aumento na globulina ligadora de hormônios sexuais (SHBG) $(19,20)$.

A acidose metabólica é também reconhecida como uma das causas do atraso do crescimento, como demonstrado em pacientes com acidose tubular não-tratada. A acidose pode contribuir atuando no eixo GH/IGF, reduzindo a secreção do GH e os níveis de IGF-1. Há estudos demonstrando também que a acidose causa resistência aos efeitos anabólicos do $\mathrm{GH}$, suprime a síntese de albumina e promove o catabolismo protéico $(2,10)$.

O crescimento pode ser também comprometido pelo desenvolvimento da osteodistrofia renal. Esta engloba uma gama de desordens, desde o alto turnover ósseo, em razão do hiperparatireoidismo secundário, até a osteomalacia e a adinamia óssea. O hiperparatireoidismo secundário pode comprometer o crescimento modulando genes envolvidos na formação de osso 
endocondral e alterando a arquitetura da placa de crescimento. Outras conseqüências da osteodistrofia renal são a redução na mineralização óssea, o aumento no número de fraturas e as deformidades ósseas. A importância da osteodistrofia renal pode ser enfatizada com base em sua incidência em crianças com DRC: $22 \%$ nos estágios 2 e 3, 38\% nos estágios 3 e 4 e quase $90 \%$ em crianças em diálise $(21,22)$.

\section{Alterações no eixo GH/IGF}

O eixo GH/IGF é importante regulador do crescimento e do metabolismo e, portanto, pacientes com DRC apresentam anormalidades em tal eixo. Como apresentado na Figura 1A, a produção e a secreção normais do hormônio de crescimento $(\mathrm{GH})$ pela hipófise anterior são estimuladas positivamente pelo hormônio liberador da secreção de $\mathrm{GH}(\mathrm{GHRH})$ e negativamente pela somatostatina (SRIF), sendo contra-reguladas negativamente pelas concentrações séricas de GH e de insulin-like growth factor I (IGF-1). A secreção do GH também é estimulada pela ghrelina, que é secretada pelo estômago e pelo hipotálamo e está possivelmente envolvida na regulação nutricional do eixo GH/IGF. O GH circulante estimula a produção e a liberação de IGF-1, primariamente no fígado. A maior parte da IGF-1 circulante encontra-se como complexo ternário ligada com a insulin-like growth factor binding protein 3 (IGFBP-3) e com a subunidade ácidolábil (ALS), e menor quantidade está associada a outras IGFBPs. Menos de $1 \%$ apresenta-se em estado livre ativo, mediando muitos dos efeitos biológicos do GH. O GH também apresenta efeitos diretos em diversos tecidos, incluindo os ossos $(5,10)$.

As crianças com DRC apresentam concentrações elevadas de GH circulante em conseqüência do aumento da pulsatilidade de secreção e diminuição do clearance renal de excreção. Contudo, tais pacientes apresentam resposta reduzida ao GH e IGF-1 endógenos (5). Um importante mecanismo para a resistência ao GH na uremia inclui alterações do receptor de $\mathrm{GH}$ (GHR), envolvendo as vias das proteínas janus kinase 2 (JAK2) e signal transducer and activador of the transcription (STAT) (23). Tais vias devem estar funcionantes para que a expressão da IGF-1 possa ocorrer com o estímulo do GH. A redução na densidade do GHR em órgão-alvo também exerce efeito na resistência ao $\mathrm{GH}$. Tal densidade é estimada pela concentração sérica de GH binding protein (GHBP), um subproduto da proteólise do GHR, sendo esta inversamente relacionada ao grau da DRC $(5,23-25)$.
A presença da resistência ao IGF-1 também reflete no aumento das concentrações séricas de IGFBPs, em especial a 1, 2, 4 e 6, o que acarreta redução nas concentrações de IGF-1 livre (Figura 1B). A maior proteólise da IGFBP-3 leva à redução do IGF-1, disponível para a formação do complexo ternário IGF-1/ALS/ IGFBP-3. Juntos, tais eventos, explicam resumidamente os motivos das não ações, direta ou indireta, do GH e da IGF-1 endógenos no crescimento de crianças com DRC $(5,10,23-25)$.

\section{A)}

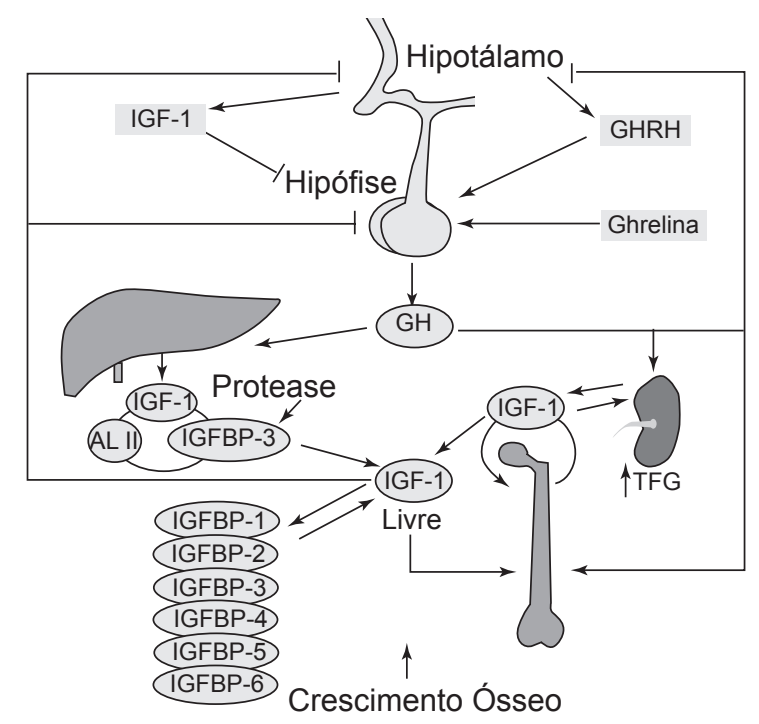

B)

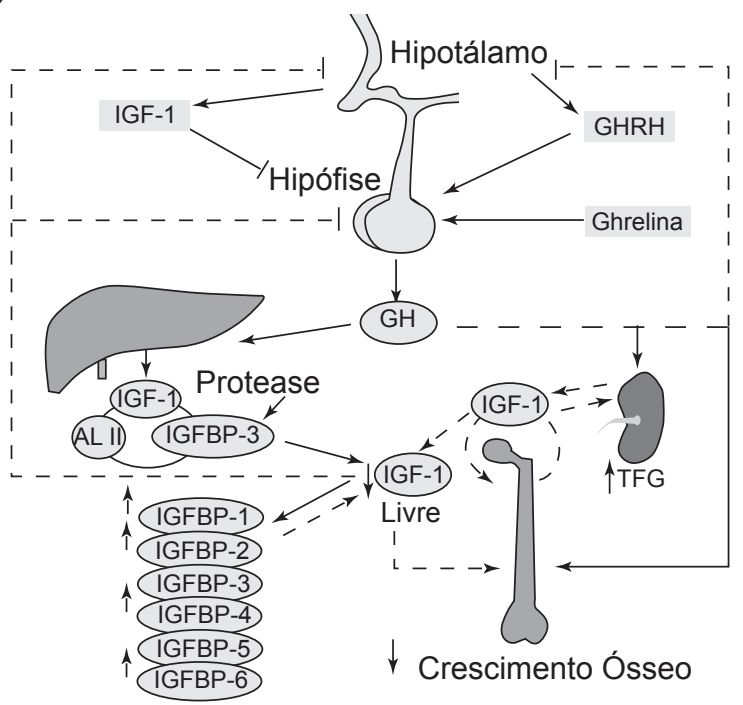

Figura 1. Eixo GH-IGF. A) fisiologia normal do eixo; B) fisiopatologia do eixo em pacientes com doença renal crônica. Adaptada de Mahan e Warady (10). 


\section{Influência do corticóide no eixo GH/IGF/IGFBP}

O atraso no crescimento é um efeito colateral da corticoterapia na infância e é mediado em parte por alterações no eixo hormonal do eixo GH-IGF e em parte por efeitos diretos do corticóide na placa de crescimento epifisária $(2,7,10)$.

A corticoterapia tem sido implicada, in vivo, como importante fator de distúrbio na espessura da placa epifisária de crescimento, no aumento da apoptose de condrócitos e na redução da expressão do vascular endothelial growth factor (VEGF) por condrócitos hipertróficos, dessa forma, reduzindo a invasão de vasos sangüíneos da metáfise na junção condro-óssea (26). Tais efeitos, provavelmente, alteram a dinâmica ossificação endocondral e assim contribuem para o atraso no crescimento induzido por corticóides.

Os mecanismos de interação entre os corticóides e os fatores de crescimento em nível celular e molecular são apenas parcialmente compreendidos. Os corticóides inibem diretamente a transcrição de IGF-1. Condrócitos murinos da placa epifisária, em modelo de cultura celular primária, tiveram seu crescimento inibido por dexametasona, em situação basal ou estimulados por GH ou IGF-1. A dexametasona também inibiu a transcrição do RNAm do receptor de GH (GHR) e reduziu a expressão do GHR, basal ou estimulado por $\mathrm{GH}$, mas não afetou a expressão do receptor de IGF-1 (IGFR). A dexametasona também inibiu a estimulação da secreção local e ação parácrina do IGF-1, bem como a expressão homóloga do IGFR e GHR (27).

Os hormônios calciotrópicos paratormônio (PTH) e 1,25-diidroxivitamina $\mathrm{D}\left(\mathrm{l} \alpha, 25(\mathrm{OH})_{2} \mathrm{D}_{3}\right)$ aumentaram a proliferação celular por meio da estimulação da secreção parácrina de IGF-1. Assim, não é de se surpreender que os corticóides inibam o crescimento celular induzido por PTH, bem como por $1 \alpha, 25(\mathrm{OH})_{2} \mathrm{D}_{3}(28)$.

Na DRC, os níveis de IGFBP séricos aumentam com o grau do quadro. Concomitantemente, os condrócitos da placa de crescimento exercem importante ação autócrina e parácrina secretando IGFBPs. Assim, as IGFBPs não apenas regulam a ação das IGFs na circulação como, também, em nível celular (7). A estimulação com IGF-1 ou IGF-2 pode aumentar o RNAm de IGFBP- 5 cerca de 11 vezes, enquanto o RNAm de outras IGFBPs pode aumentar apenas levemente. A dexametasona suprimiu a IGFBP-5 em cerca de 70\%, mas, ao mesmo tempo, induziu a expressão do dobro de IGFBP-3. Entre as complicadas e diferentes atividades das IGFBPs, mostrou-se que a IGFBP-5 aumenta a atividade mitogênica em di- versos tipos celulares, enquanto a IGFBP-3 age como inibidor de crescimento. Assim, os efeitos diferenciais da dexametasona no padrão das IGFBPs pode contribuir para o atraso no crescimento induzido por corticóides $(29,30)$. Doses suprafisiológicas do GH e/ou do IGF-1 podem parcialmente superar os efeitos inibidores do crescimento dos corticóides nas placas de crescimento. As concentrações séricas de IGFBP3 e IGFBP-5 aumentam durante o tratamento com hrGH $(4,7,31,32)$. In vitro, o GH estimula a síntese de IGF e a secreção de IGFBP-5 induzida por IGF em células-alvo $(28,32)$. O IGF-l aumenta a produção de colágeno tipo I em osteoblastos humanos tratados com hidrocortisona (33). O complexo de alterações induzidas pela corticoterapia no eixo GH/IGF/IGFBP está resumido na Figura 2.

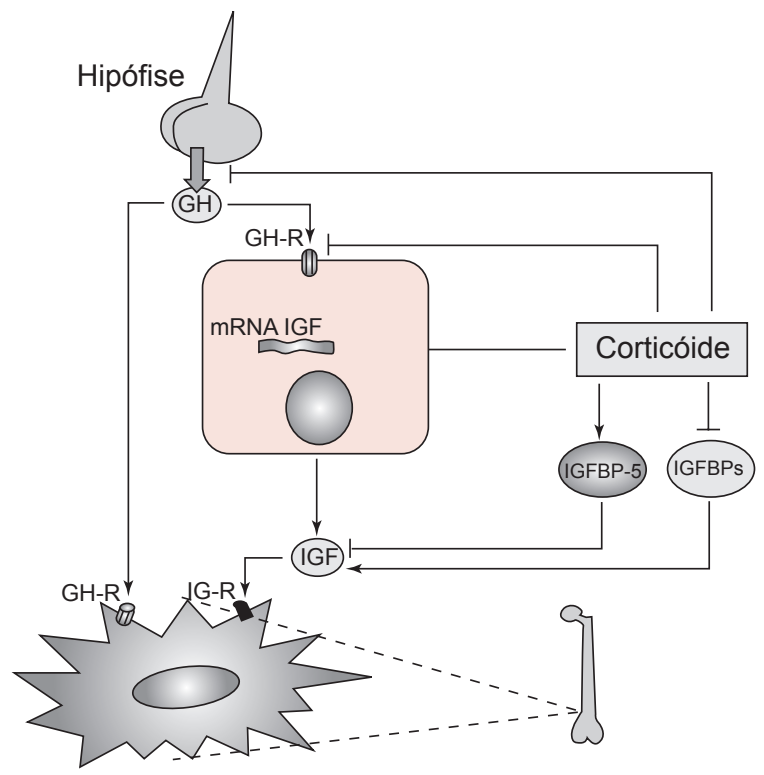

Figura 2. Influências dos corticóides no eixo GH-IGF. Adaptada de Ulinski e Cochat (7).

Os efeitos negativos dos corticóides no crescimento de pacientes com DRC córtico-dependente ou transplantados são ressaltados quando a estratégia terapêutica substitui os corticóides pela ciclosporina ou pelo tacrolimo, com melhora significativa no crescimento $(34,35)$.

\section{TRATAMENTO DO ATRASO NO CRESCIMENTO NA DOENÇA RENAL CRÔNICA}

A NFK recomenda a correção das deficiências nutricionais e das alterações metabólicas existentes antes de se considerar o tratamento com hrGH. Os ajustes ou a su- 
plementação na dieta devem ser realizados em pacientes que apresentem ingestão insuficiente de calorias, proteínas ou outros nutrientes. Também, recomenda-se a normalização do metabolismo ácido-básico. É necessário o máximo controle do metabolismo do cálcio e do fósforo séricos antes de iniciar a terapia com hrGH, devendo-se manter a fosfatemia em, no máximo, 1,5 vezes acima do limite para a idade, mas também estando alerta para a correção dos casos com hipofosfatemia (1). Em pacientes transplantados, as terapias sem corticóides minimizam o atraso no crescimento $(34,35)$.

\section{Eficácia do tratamento com hormônio de crescimento em crianças com doença renal crônica}

Tendo em vista as altas concentrações de GH na DRC, o uso do hrGH não foi cogitado até os trabalhos de Mehls e Ritz (36), em 1983, que demonstraram aumento no comprimento e no peso de ratos urêmicos tratados com GH suíno. Desde então, vários outros estudos foram publicados demonstrando aumento na velocidade de crescimento com altas doses de hrGH. A metanálise publicada recentemente, com a seleção de 15 estudos com 629 crianças, concluiu que: a) um ano de uso de hrGH $\left(0,35 \mathrm{mg} / \mathrm{kg} / \mathrm{semana}\right.$ ou $28 \mathrm{UI} / \mathrm{m}^{2} /$ semana $)$ aumentou a velocidade de crescimento em $3,8 \mathrm{~cm} /$ ano em comparação aos pacientes não-tratados com hrGH; b) os estudos foram, em sua maioria, de curta duração e não permitiram concluir o efeito deste tratamento na altura final dos pacientes com DRC (8).

Foram descritos resultados semelhantes por Mehls e cols. (36) ao revisarem os dados do sistema KIGS (Kabi, atualmente Pfizer, International Growth Study) com 1.700 crianças em todos os estágios de DRC, inclusive pacientes transplantados. A idade média de início do hrGH foi de $9,1 \pm 4,4$ anos com idade óssea atrasada em cerca de dois anos em média. Apenas 20\% dos pacientes eram pré-púberes no momento de início do hrGH e $70 \%$ do gênero masculino. $\mathrm{O}$ z-escore médio da estatura era de $-2,5 \pm 1,2$. A dose de hrGH utilizada variou de $0,30 \mathrm{mg} / \mathrm{kg} / \operatorname{semana}\left(24 \mathrm{UI} / \mathrm{m}^{2} /\right.$ semana) a $0,35 \mathrm{mg} / \mathrm{kg} / \operatorname{semana}\left(28 \mathrm{UI} / \mathrm{m}^{2} /\right.$ semana $)$. Em geral, os pacientes apresentaram boa resposta ao hrGH nos primeiros dois anos de tratamento, com melhor resposta nos que iniciaram mais cedo o hrGH e, portanto, puderam usar por mais tempo o tratamento.

São poucos os estudos com tratamento de longa duração com hrGH nos quais os pacientes com DRC tenham alcançado a altura final. Em 2001, Haffner e Schaefer (37) revisaram 11 estudos em que os pacientes com DRC tratados com hrGH atingiram a altura final. Eles concluíram que o benefício adquirido com o uso prolongado de hrGH atingiu ganho na altura final de 1,0 a 1,5 desvio-padrão. Desde este estudo publicado em 2001, mais outros dois estudos com altura final em pacientes com DRC e uso de hrGH foram publicados $(38,39)$ com resultados semelhantes. Em 2003 (40), outro estudo mostrou que pacientes com DRC sem uso de hrGH mostra comprometimento importante da altura final na maioria dos casos.

Os pacientes com DRC após o transplante podem retomar espontaneamente o crescimento normal ou não. Por este motivo, a maioria dos serviços tende a observar a velocidade de crescimento após um ano do transplante e somente após esta avaliação decidir pela (re)introdução do hrGH (41). Guest e cols. (42) avaliaram 90 pacientes transplantados que apresentaram enxertos funcionantes um ano após o transplante. Destes, 46 foram tratados com hrGH e 44 não. Após um ano de acompanhamento, observaram melhora significativa na velocidade de crescimento do grupo tratado $(4,1 \mathrm{~cm} /$ ano para $7,7 \mathrm{~cm} / \mathrm{ano})$, o que não ocorreu no grupo não-tratado, sem aparecimento de efeitos colaterais significativos, o que justificou a inclusão de todos no tratamento com hrGH.

Fine e cols. (43), avaliando 68 pacientes transplantados tratados com hrGH durante um ano, também mostraram ganho em altura quando comparados a controles não-tratados (z-escore após um ano de tratamento com hrGH: $0,49 \pm 0,1$ versus $-0,10 \pm 0,8)$. Em análise mais recente dos dados da base NAPTRCS, Fine e cols. (44) apontaram que a altura final foi significativamente melhor nos pacientes transplantados que receberam $\mathrm{hrGH}(\mathrm{n}=$ 513 ), em relação ao grupo que não recebeu hrGH após o transplante $(\mathrm{n}=2.263)$ ( $\mathrm{z}$-escore da altura final: $-1,83 \pm$ $0,14$ versus $-2,60 \pm 0,05)$. A comparação entre os dois grupos também mostrou não haver diferenças na incidência de rejeição ao enxerto ou perda do transplante.

\section{Segurança do tratamento com hormônio de crescimento}

Utilizando a base de dados do NAPTRCS, Fine e cols. (43), avaliando pacientes com DRC, em diálise ou transplantados que usaram hrGH por mais de seis anos em relação aos que não usaram hrGH, compararam a freqüência de efeitos adversos relacionados ao hrGH e não observaram diferença significativa na incidência de câncer, subluxação de epífise femural, necrose avascular da cabeça do fềmur, intolerância à glicose, pancreatite, deterioração da função renal, rejeição aguda de enxerto, retenção de líquidos e hipertensão intracraniana benigna. 


\section{Dose do hormônio de crescimento}

A metanálise encontrou diferença significativa maior na dose de $0,30\left(28 \mathrm{UI} / \mathrm{m}^{2} /\right.$ semana $)$ em relação a $0,17 \mathrm{mg} /$ $\mathrm{kg} / \mathrm{semana}$ (14 UI $/ \mathrm{m}^{2} /$ semana) utilizada para pacientes com DRC com atraso no crescimento. Em ambos os grupos, não se observou avanço significativo da idade óssea, durante o primeiro ano de tratamento, em comparação a pacientes não-tratados ou que usaram placebo. Em geral, a dose recomendada de hrGH para pacientes com DRC, em tratamento conservador, em diálise ou pós-transplante é de 0,30 a $0,35 \mathrm{mg} / \mathrm{kg} / \mathrm{semana}\left(24\right.$ a $28 \mathrm{UI} / \mathrm{m}^{2} / \mathrm{sema}^{-}$ na), via subcutânea, administrada diariamente à noite (2).

\section{ALGORÍTIMO PROPOSTO PARA AVALIAÇÃO E TRATAMENTO DO ATRASO NO CRESCIMENTO DE CRIANÇAS CÖM DOENÇA RENAL CRÔNICA}

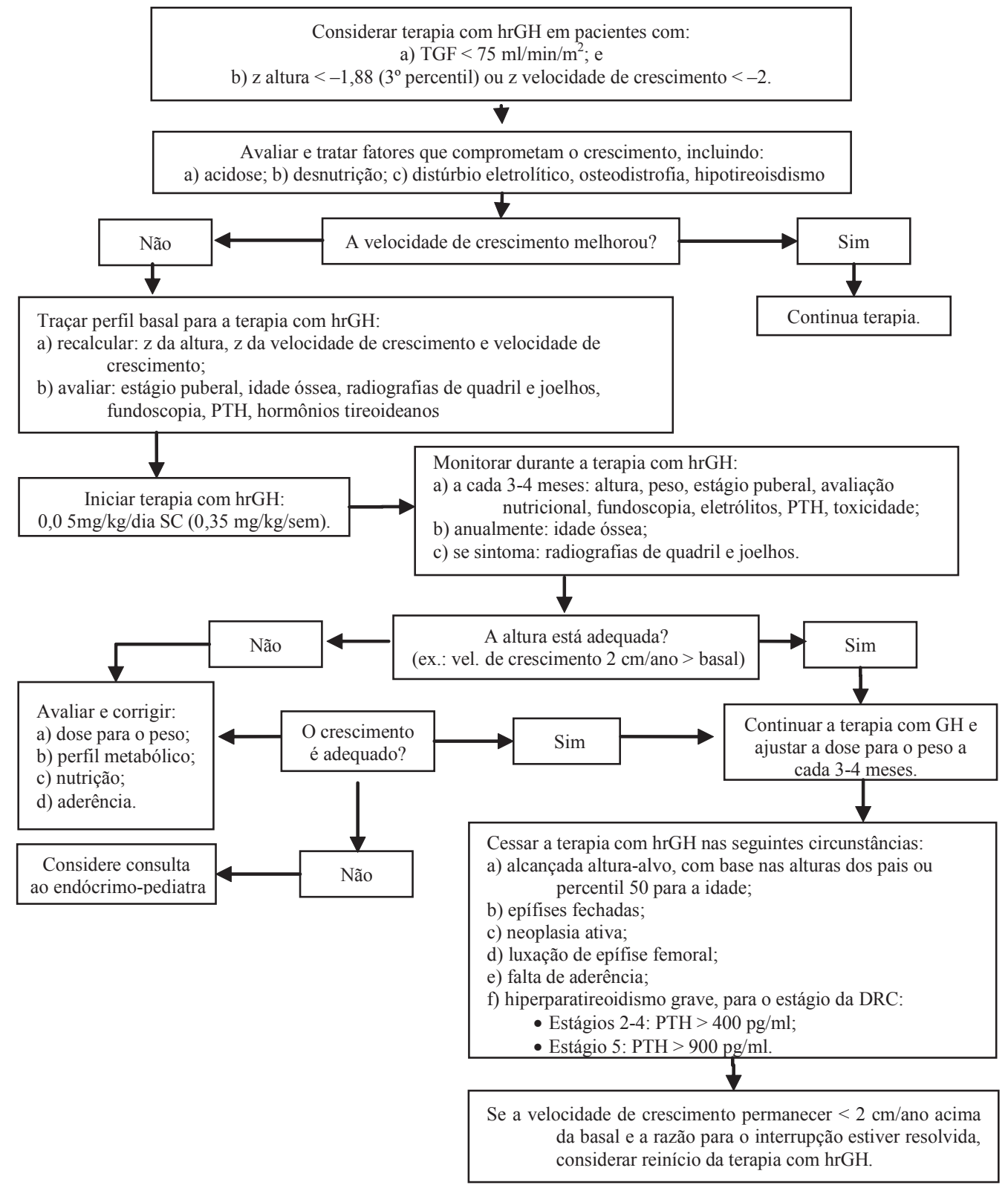

Algoritmo proposto por Mahan e Warady (10) para avaliação e tratamento do atraso no crescimento de crianças com doença renal crônica. 


\section{CONCLUSÕES}

Os estudos publicados até o momento com o uso de hrGH em pacientes com atraso no crescimento e DRC, em tratamento conservador, dialítico ou pós-transplante, permitem concluir-se que se trata de opção terapêutica segura e com efeitos benéficos no crescimento quanto mais precoce for sua introdução e quanto maior tempo for a sua utilização. A eficácia terapêutica do hrGH depende também do tratamento adequado de todas as alterações nutricionais, metabólicas e hormonais associadas à DRC.

\section{REFERÊNCIAS}

1. National Kidney Foundation. K/DOOI clinical practice guidelines for chronic kidney disease: evaluation, classification, and stratification. Am J Kidney Dis. 2002;39(2 Suppl 1):S1-266.

2. Chesney RW, Brewer E, Moxey-Mims M, Watkins S, Furth SL, Harmon WE, et al. Report of NIH task force on research priorities in chronic kidney disease in children. Pediatr Nephrol. 2006;21:14-25.

3. NAPRTCS Annual Report. [acesso em 2005]. Disponível em: https://web.emmes.com/study/ped/annlrept/annlrept2005.pdf

4. Seikaly MG, Salhab N, Warandy BA, Stablein D. Use o hrGH in children with chronic kidney disease: lessons from NAPRTCS. Pediatr Nephrol. 2007;22:1195-204.

5. Mahesh S, Kaskel F. Growth hormone axis in chronic kidney disease. Pediatr Nephrol. 2008;23:41-8.

6. Gorman G, Frankenfield D. Linear growth in pediatric hemodialysis patients. Pediatr Nephrol. 2008;23:123-7.

7. Ulinski $T$, Cochat P. Longitudinal growth in children following kidney transplantation: from conservative to pharmacological strategies. Pediatr Nephrol. 2006;21:903-9.

8. Vimalanchandra D, Hodson EM, Willis NS, Craig JC, Cowell C, Knight JF. Growth hormone for children with chronic kidney disease (Cochrane Review). Cochrane Database Syst Rev. 2006;19:CD003264.

9. Williams R. Growth hormone underutilized in pediatric hemodialysis population. Nat Clin Pract Nephrol. 2006;2. Disponível em: doi:10.1038/ncpneph0081.

10. Mahan JD, Warady BA. Assessment and treatment of short stature in pediatric patients with chronic kidney disease: a consensus statement. Pediatr Nephrol. 2006;21:917-30.

11. Lewis M, Shaw J, Reid C, Evans J, Webb N, Verrier-Jones K. Growth in children with established renal failure: a registry analysis. Nephrol Dial Transplant. 2007;22:76-180.

12. Tom A, McCauley L, Bell L, Rodd C, Espinosa P, Yu G, et al. Growth during maintenance hemodialysis: impact of enhanced nutrition and clearance. J Pediatr. 1999;134:464-71.

13. Roth KS, Duncan LL, Chan JC. The role of nutrition in chronic renal insufficiency of childhood: how much do we know? Crit Rev Food Sci Nutr. 2005;45:259-63.

14. Jarkovská Z, Hodková M, Sazamová M, Rosická M, DusilováSulková S, Marek J, et al. Plasma levels of active and total ghrelin in renal failure: a relationship with GH/IGF-1 axis. Growth Horm IGF Res. 2005;15:369-76.
15. Mak RH, Cheung W, Cone RD, Marks DL. Orexygenic and anorexygenic mechanisms in the control of nutrition in chronic kidney disease. Pediatr Nephrol. 2005;20:427-31.

16. Rees L, Shaw W. Nutrition in children with CRF and on dialysis. Pediatr Nephrol. 2007;22:1689-702.

17. Graf L, Candelaria S, Doyle M, Kaskel F. Nutrition assessment and hormonal influences on body composition in children with chronic kidney disease. Adv Chronic Kidney Dis. 2007;14:215-23.

18. Uauy RD, Hogg RJ, Brewer ED, Reisch JS, Cunningham C, Holliday MA. Dietary and growth in infants with chronic renal insufficiency: a report from the Southwest Pediatric Nephrology Study Group and the University of California, San Francisco. Pediatr Nephrol. 1994;8:45-50.

19. Lane PH. Puberty and chronic kidney disease. Adv Chronic Kidney Dis. 2005;12:372-7.

20. Hokken-Koelega AC, Saenger P, Cappa M, Greggio N. International Workshop on Management of Puberty for Optimum Auxological Results. Unresolved problems concerning optimal therapy of puberty in children with chronic renal diseases. $J$ Pediatr Endocrinol Metab. 2001;14(Suppl 2):945-52.

21. Langman CB. Renal osteodystrophy: a pediatric perspective, 2005. Growth Horm IGF Res. 2005;15(Suppl A):42-7.

22. Santos $F$, Carbajo-Pérez $E$, Rodríguez J, Fernández-Fuente $M$, Molinos I, Amil B, et al. Alterations of the growth plate in chronic renal failure. Pediatr Nephrol. 2005;20:330-4.

23. Rabkin R, Sun DF, Chen Y, Tan J, Shaefer F. Growth hormone resistance in uremia: a role of impaired JAK/STAT signaling. Pediatr Nephrol. 2005;20:313-8.

24. Roelfsema V, Clark RG. The growth hormone and insulin-like growth factor axis: its manipulation for the benefit of growth disorders in renal failure. J Am Soc Nephrol. 2001;12: 1297-306.

25. Tonshoff B, Kiepe D, Ciarmatori S. Growth hormone/insulinlike growth factor system in children with chronic renal failure. Pediatr Nephrol. 2005;20:279-89.

26. Smink JJ, Buchholz IM, Hamers N, van Tilburg CM, Christis C, Sakkers RJ, et al. Short-term glucocorticoid treatment of piglets causes changes in growth plate morphology and angiogenesis. Osteoarthritis Cartilage. 2003;11:864-71.

27. Jux C, Leiber K, Hügel U, Blum W, Ohlsson C, Klaus G, et al. Dexamethasone impairs growth hormone $(\mathrm{GH})$-stimulated growth by suppression of local insulin-like growth factor (IGF)I production and expression of GH- and IGF-I-receptor in cultured rat chondrocytes. Endocrinology. 1998;139:3296-305.

28. Klaus G, Jux C, Fernandez P, Rodriguez J, Himmele R, Mehls O. Suppression of growth plate chondrocyte proliferation by corticosteroids. Pediatr Nephrol. 2000;14:612-5.

29. Koedam JA, Hoogerbrugge CM, van Buul-Offers SC. Differential regulation of IGF-binding proteins in rabbit costal chondrocytes by IGF-I and dexamethasone. J Endocrinol. 2000;165:557-67.

30. Smink JJ, Koedam JA, Koster JG, van Buul-Offers SC. Dexamethasone-induced growth inhibition of porcine growth plate chondrocytes is accompanied by changes in levels of IGF axis components. J Endocrinol. 2002;174:343-52.

31. Oliveira JC, Machado Neto FA, Morcilo AM, Oliveira LC, Belangero VMS, Gelonese Neto B, et al. Insuficiência renal crônica e hormônio de crescimento: efeitos no eixo GH-IGFe na leptina. Arq Bras Endocrinol Metab. 2005;49: 964-70.

32. Mehls O, Himmele R, Hömme M, Kiepe D, Klaus G. The interaction of glucocorticoids with the growth hormone-insulin- 
like growth factor axis and its effects on growth plate chondrocytes and bone cells. J Pediatr Endocrinol Metab. 2001;14(Suppl 6):1475-82.

33. Jonsson KB, Ljunghall S, Karlström O, Johansson AG, Mallmin $\mathrm{H}$, Ljunggren $\mathrm{O}$. Insulin-like growth factor I enhances the formation of type I collagen in hydrocortisone-treated human osteoblasts. Biosci Rep. 1993;13:297-302.

34. Klare B, Strom TM, Hahn H, Engelsberger I, Meusel E, Illner WD, et al. Remarkable long-term prognosis and excellent growth in kidney-transplant children under cyclosporine monotherapy. Transplant Proc. 1991;23:1013-17.

35. Shampiro R, Scantlebury VP, Jordan ML, Vivas C, Ellis D, Lombardozzi-Lane $\mathrm{S}$, et al. Pediatric renal transplantation under tacrolimus-based immunosuppression. Transplantation. 1999;67:299-303.

36. Mehls O, Ritz E. Skeletal growth in experimental uremia. Kidney Int. 1983;21(Suppl):53-62.

37. Haffner D, Schaefer F. Does recombinant growth hormone improve adult height in children with chronic renal failure? Semin Nephrol. 2001;21:490-7.

38. Fine RN, Stablein D. Long-term use of recombinant growth hormone in pediatric allograft recipients: a report from the NAPTRCS Transplant Registry. Pediatr Nephrol. 2005;20:404-8.

39. Crompton $\mathrm{CH}$. Australian and New Zeland Paediatric Nephrology Association. Long-term recombinant human growth hormone use in Australian children with renal disease. Nephrology (Carlton). 2004;9:325-30.

40. Andre JL, Bourquard R, Guillemin F, Krier MJ, Briancon S. Final height in children with chronic renal failure who have not received growth hormone. Pediatr Nephrol. 2003;18:685-9.
41. Mehls O, Lindberg A, Nissel R, Wühl E, Schaefer F, Tönshoff B, et al. Growth hormone treatment in short children with chronic kidney disease. In: Ranke MB, Price DA, Reiter EO, editores. Growth hormone therapy in pediatrics. Basel: Karger; 2007. p. 407-21.

42. Guest G, Berard E, Crosnier H, Chevallier T, Rappaport R, Broyer M. Effects of growth hormone in short children after renal transplantation. French Society of Pediatric Nephrology. Pediatr Nephrol 1998;12:437-46.

43. Fine RN, Ho M, Tejani A, Blethen S. Adverse events with hrGH treatment of patients with chronic renal insufficiency and endstage renal disease. J Pediatr. 2003;142:539-45.

44. Fine RN, Stablein D, Cohen AH, Tejani A, Kohaut E. Recombinant human growth hormone post-renal transplantation in children: a randomized controlled study of the NAPRTCS. Kidney Int. 2002;62:688-96.

\section{Endereço para correspondência:}

\section{Gil Guerra-Júnior}

Departamento de Pediatria, Faculdade de Ciências

Médicas, Unicamp

CP 6111

13083-970 Campinas, SP

E-mail: gilguer@fcm.unicamp.br 